Ann. Riol. anim. Bioch. Biophys., 1979, 19 (2 B), 537-541.

\title{
B. Effets d'une surcharge alimentaire en huile de colza sur l'activité électrique du cœur du rat
}

\author{
par E. DEROUBAIX, E. CORABGUF
}

Laboratoire de Physiologie Comparée ef de Physiologie Cellulaire associé au CNRS Université Paris XI, 91405 Orsay Cedex.

Summary. Effects of dietary overload of rapeseed oil on cardiac electrical activity in rats.

Ventricular electrical activity was recorded in isolated perfused rat heart with conventional floating microelectrodes. Two groups of animals were studied, one fed for 2 weeks with peanut oil (control group) and the other with Primor rapeseed oil (test group). The parameters measured were resting potential, action potential amplitude and duration (at 80 p. 100 repolarization) and maximal rate of rise of the action potential upstroke $\left(\mathrm{dV} / \mathrm{dt}_{\max }\right)$. No significant differences were observed between the control and test groups, in spite of the marked cellular accumulation of lipid droplets observed by other authors in the rat heart after 2 weeks of Primor rapeseed oil intake.

L'activité électrique transmembranaire cardiaque (Corabœuf, 1971, 1978) est due à l'ouverture successive de plusieurs conductances ioniques passives, à savoir une conductance sodique rapide, une conductance calcicosodique lente, et une conductance potassique très lente. Les deux premières donnent naissance à un courant entrant et sont dépolarisantes : elles sont responsables respectivement de la pointe et du plateau du potentiel d'action cardiaque. La troisième donne naissance à un courant sortant et est repolarisante : elle favorise la terminaison du potentiel d'action. Au repos, les membranes cardiaques présentent une perméabilité dominante au potassium, mais celle-ci, qui décroît au cours du potentiel d'action, est sans doute différente de celle qui s'ouvre progressivement au cours du plateau. Les systèmes de transports actifs électrogènes participent sans doute aussi au développement de l'activité électrique cardiaque.

On sait depuis longtemps que les altérations métaboliques entraînent en général un raccourcissement du plateau du potentiel d'action (Trautwein et al., 1954 ; Corabœuf ef al., 1958) avec ou sans dépolarisation membranaire diastolique. Ce raccourcissement peut être attribué soit à une augmentation d'un courant sortant, soit à une diminution d'un courant entrant. L'idée qu'une diminution du courant entrant lent calciosodique est responsable des altérations électriques d'origine métabolique a récemment gagné du terrain (Mc Donald ef Mc Leod, 1973 ; Schneider et Sperelakis, 1974 ; Kohlhardt ef Kübler, 1975 ; Nargeot, 1976). Cette diminution pourrait être due à une moindre production d'ATP et $\mathrm{AMP}_{\mathrm{c}}$ par les cellules cardiaques. 
Une accumulation importante de lipides à l'intérieur des cellules (Bodak, 1972 ; Vodovar ef Desnoyers, 1974 ; Engfeldt et Brunius, 1975 ; Hatt et Bodak, 1975) pouvant a priori altérer le fonctionnement cellulaire par lésion mitochondriale, atteinte des réserves en glycogène ou interaction avec l'une ou l'autre des étapes du métabolisme, il n'est pas exclu à priori qu'une telle accumulation puisse exercer une action sur l'activité électrique transmembranaire, en particulier au niveau du plateau du potentiel d'action.

Nous avons en conséquence analysé les effets d'une surcharge en huile de colza sur le potentiel de membrane diastolique et sur les diverses phases du potentiel d'action ventriculaire du cœur de rat isolé et perfusé.

\section{Matériel et méthodes.}

L'activité électrique cellulaire (potentiel diastolique ou de repos, PR et potentiel d'action, PA) a été enregistrée au niveau du ventricule du cœur isolé et perfusé de rat. Deux groupes de 6 animaux fournis par $G$. Durand de la Station de Recherches de Nutrifion de l'INRA et pesant 150 à $200 \mathrm{~g}$ ont été utilisés : l'un a reçu de l'huile de colza riche en acide érucique (15 p. 100 du régime), l'autre une quantité égale d'huile d'arachide. Ce régime a été distribué pendant 3 semaines.

Les animaux sont assommés, le cour rapidement prélevé est placé dans une cuve expérimentale où il esł perfusé à pression constante $(70 \mathrm{~cm}$ d'eau) par voie aortique (technique de Langendorff). La température du liquide physiologique de perfusion a été maintenue à $25^{\circ} \mathrm{C}$, température à laquelle le décours temporel du PA de rat s'avère particulièrement sensible à la plupart des agents (Corabœuf, 1960). La composition du liquide de perfusion est en $\mathrm{mM}, \mathrm{NaCl}: 130 ; \mathrm{KCl}: 5,6 ; \mathrm{PO}_{4} \mathrm{HNa}: 0,6$; $\mathrm{CO}_{3} \mathrm{HNa}: 11,9: \mathrm{MgCl}_{2}: 1,1 ; \mathrm{CaCl}_{2}: 2,1 ;$ glucose : 11 . La solution est saturée par un mélange gazeux de 95 p. $100 \mathrm{O}_{2}-5$ p. $100 \mathrm{CO}_{2}$; le pH est de 7,4. L'activité électrique est enregistrée par microélectrodes flottantes (résistance de 15 à $10 \Omega \mathrm{M}$ ) en différentes régions du ventricule gauche. 15 à 25 pénétrations cellulaires sont effectuées en des points différents pour chaque animal et les différents paramètres mesurés ( $P R$, amplitude maximale du PA, vitesse maximale de sa phase ascendante $\left(\mathrm{dV} / \mathrm{dt}_{\max }\right)$, durée du PA à 80 p. 100 de repolarisation (voir fig. 1). Il est connu, ef la présente expérience l'a confirmé, qu'il n'existe normalement que très peu de différence entre les PA des diverses régions de l'épicarde gauche. Les moyennes ont donc été faites pour chaque cœur à partir de la totalifé des mesures.

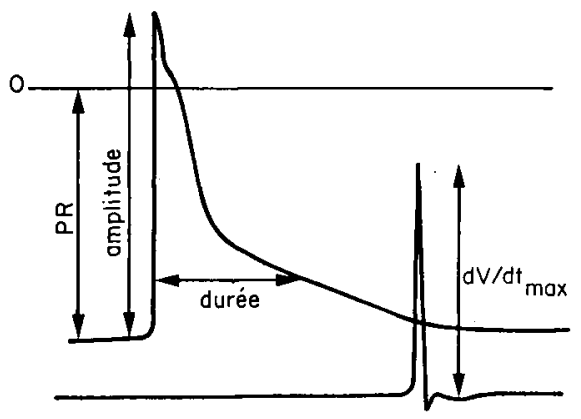

FIG. 1. - Schéma d'un potentiel d'action ventriculaire de rat d $24^{\circ} \mathrm{C}$ avec indication des différents paramètres mesurés. PR : Potentiel de repos ; $\mathrm{dV} / \mathrm{dt}_{\max }:$ vitesse maximale de la phase ascendante du PA. 


\section{Résultats.}

La fréquence spontanée des cœurs isolés a été mesurée au début de chaque expérience. II n'est pas apparu de différence significative de cette fréquence entre les animaux traités et les animaux témoins. Néanmoins toułes les mesures d'activité électrique cellulaire ont été effectuées sur les cœurs entraînés électriquement à fréquence constante $(F=2 \mathrm{~Hz}$; durée de chaque stimulus : $2 \mathrm{~ms}$; amplitude ajustée à 125 p. 100 du seuil).
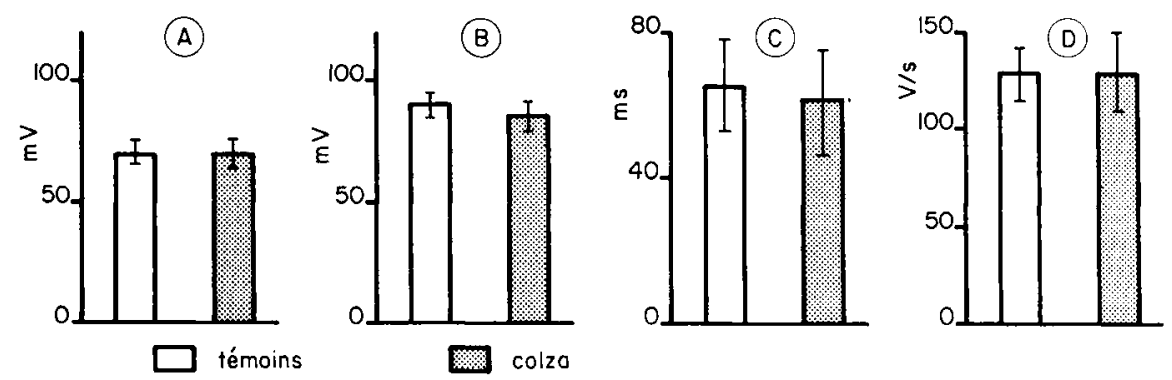

FIG. 2. - Histogramme résumant les valeurs des différents paramètres mesurés dans les deux groupes d'animaux. A : potentiel de repos en $\mathrm{mV}$; $B$ : amplitude du PA en $\mathrm{mV} ; C$ : durée en ms mesurée à 80 p. 100 de repolarisation : $D$ : vifesse maximale de la phase ascendante du potentiel d'action en $\mathrm{V} / \mathrm{s}$.

La comparaison des mesures effectuées dans le groupe témoins et dans le groupe des rats traités à l'huile de colza a donné respectivement pour le potentiel de repos (PR) $68,7 \pm 3,6 \mathrm{mV}$ ef $68,2 \pm 4,7 \mathrm{mV}$, pour l'amplitude du PA $89,5 \pm 5,6 \mathrm{mV}$ ef $83,1 \pm 6,5 \mathrm{mV}$; pour la durée du plateau $68,4 \pm 14,7 \mathrm{~ms}$ et $65,3 \pm 16,3 \mathrm{~ms}$ et pour la vitesse maximale de la phase ascendante du potentiel d'action $128,2 \pm 11,2 \mathrm{~V} / \mathrm{s}$ ef $126,2 \pm 19,1 \mathrm{~V} / \mathrm{s}$. La figure 2 résume l'ensemble de ces résultats. La figure 3 donne un exemple de PA obtenus chez un animal traité et chez un animal témoin.

\section{Conclusion.}

Ces résultats montrent qu'il est impossible de mettre en évidence, à ce stade du régime (21 jours), aucune différence significative entre les manifestations électriques cellulaires cardiaques (ventricule gauche) des rats recevant de l'huile de colza ef des rats recevant de l'huile d'arachide. Il est intéressant de constater qu'une surcharge cellulaire en lipides aussi imporiante que celle qui apparaît entre les $7^{\mathrm{e}}$ et $15^{\mathrm{e}}$ jours, à la suite de l'ingestion d'huile de colza, n'entraîne cependani aucune altération décelable des perméabilités ioniques membranaires. 

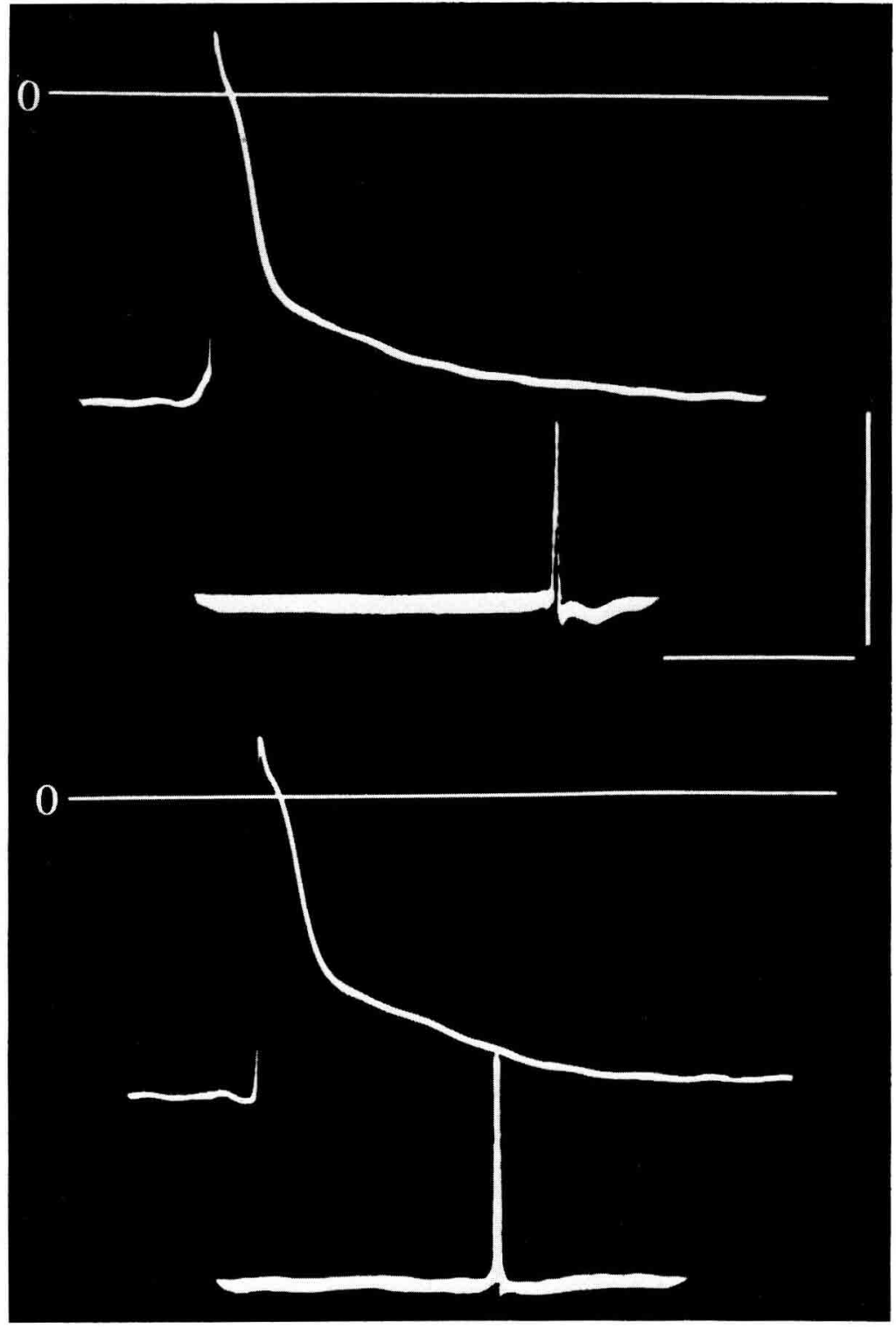

FIG. 3. - Potentiel d'action ventriculaire (tracé supérieur) et vifesse maximale de la phase ascendante (tracé inférieur) enregistrés en haut sur un raf témoin, en bas sur un rat ayant reçu de l'huile de colza. Echelle horizontale : $50 \mathrm{~ms}$; échelle verticale $50 \mathrm{mV}$ et $150 \mathrm{~V} / \mathrm{s}$. 


\section{Références}

BODAK A., 1972. Etude au microscope électronique des lésions cellulaires myocardiques induites par une surcharge en huile de colza chez le rat. Thèse Doct. Méd., Fac. Méd. Cochin-Port-Royal, Paris.

CORABCEUF E., 1960. Aspects cellulaires de l'électrogenèse cardiaque chez les vertébrés. J. Physiol. Paris, 52, 326-417.

CORABCEUF E., 1971. Courants ioniques transmembranaires dans les cellules cardiaques : applications à la pharmacologie. Actual. Pharmacol., 24, 153-182.

CORABCEUF E., 1978. Ionic basis of electrical activity in cardiac tissues. Amer. J. Physiol., 234, 101-116.

CORABCEUF E., GARGOUIL Y. M., LAPLAUD J., DESPLACES A., 1958. Action de l'anoxie sur les potentiels électriques des cellules cardiaques de mammifères actives ef inertes (tissu ventriculaire isolé de cobaye). C. R. Acad. Sci., 246, 3100-3103.

ENGFELDT B., BRUNIUS E., 1975. Morphological effects of rapeseed oil in rats. Acta med. scand., 585 (suppl.), $15-40$.

HATT P. Y., BODAK A., 1975. Myocardial lesions induced by rapeseed oil rich diet in the rat : ultrastructural aspects. In FLECKENSTEIN A., RONA G., Recent Adv. Studies Cardiac Structure Metab., 6, 479-486. Univ. Park Press, Baltimore.

KOHLHARDT M., KUBLER M., 1975. The influence of metabolic inhibitors upon the transmembrane slow inward current in the mammalian ventricular myocardium. Naunyn Schmiedeberg's Arch. Pharmacol., 290, 265-274.

Mc DONALD T. F., Mc LEOD D. P., 1973. Metabolism and the electrical activity of anoxic ventricular muscle. J. Physiol. Lond., 229, 559-582.

NARGEOT J., 1976. Current clamp and voltage clamp study of the inhitory action of DNP on membrane electrical properties of frog auricular heart muscle. J. Physiol. Paris, 72, 171-180.

SCHNEIDER J. A., SPERELAKIS N., 1974. The demonstration of energy dependence of the isoproterenol-induced transcellular $\mathrm{Ca}^{2+}$ current in isolated perfused guinea pig hearts; an explanation for the mechanical failure of ischemic myocardium. J. Surg. Res., 16, 389-403.

TRAUTWEIN W., GOTTSTEIN U., DUDEL J., 1954. Der Aktionsstrom der Myokardfaser in Sauerstoffmangel. Pflügers Arch., 260, 40-60.

VODOVAR N., DESNOYERS F., 1974. Altérations ultrastructurales des cellules du myocarde de porc et de rat induites par le régime avec des huiles de colza et ses variétés Canbra et Primor, 115138. In Colloques INSERM, Les effets physiopathologiques des acides gras à chaine irès longue, Paris. 